\title{
ARTICLE \\ RNF6 promotes myeloma cell proliferation and survival by inducing glucocorticoid receptor polyubiquitination
}

\author{
Ying Ren ${ }^{1}$, Xin $\mathrm{Xu}^{1}$, Chen-yu Mao ${ }^{2}$, Kun-kun Han ${ }^{1}$, Yu-jia Xu ${ }^{1}$, Bi-yin Cao ${ }^{1}$, Zu-bin Zhang ${ }^{1}$, Gautam Sethi ${ }^{3}$, Xiao-wen Tang ${ }^{4}$ and
} Xin-liang Mao ${ }^{1,4,5}$

\begin{abstract}
RNF6, a RING-type ubiquitin ligase, has been identified as an oncogene in various cancers but its role in multiple myeloma (MM) remains elusive. In the present study we first showed that the expression levels of RNF6 in MM were significantly elevated compared with the bone marrow cells of healthy donors. Overexpression of RNF6 in LP1 and PRMI-8266 MM cell lines promoted cell proliferation, whereas knockdown of RNF6 led to apoptosis of MM cells. Furthermore, we revealed that RNF6, as a ubiquitin ligase, interacted with glucocorticoid receptor (GR) and induced its K63-linked polyubiquitination. Different from current knowledge, RNF6 increased GR stability at both endogenous and exogenous contexts. Such an action greatly promoted GR transcriptional activity, which was confirmed by luciferase assays and by the increased expression levels of prosurvival genes including Bcl-xL and $M c l-1$, two typical downstream genes of the GR pathway. Consistent with these findings, ectopic expression of RNF6 in MM cells conferred resistance to dexamethasone, a typical anti-myeloma agent. In conclusion, we demonstrate that RNF6 promotes MM cell proliferation and survival by inducing atypical polyubiquitination to GR, and RNF6 could be a promising therapeutic target for the treatment of MM.
\end{abstract}

Key words: multiple myeloma; RNF6; glucocorticoid receptor; ubiquitin-proteasome pathway; apoptosis; dexamethasone

Acta Pharmacologica Sinica (2020) 41:394-403; https://doi.org/10.1038/s41401-019-0309-6

\section{INTRODUCTION}

The really interesting new gene (RING) finger protein RNF6 belongs to the group of RING-C3H2C3 type E3 ubiquitin ligases, which identify specific substrate proteins for ubiquitination [1]. Several proteins have been identified as substrates of RNF6. As early as 2009, RNF6 was reported to mediate the K6/K27-linked polyubiquitination of androgen receptor (AR) and selectively promote its transcriptional activity, thereby inducing prostate cancer proliferation [2]. Moreover, RNF6 can lead to the polyubiquitination and degradation of transducin-like enhancer of split 3 (TLE3) [3] and the tyrosine phosphatase SHP-1 [4], thus promoting colorectal cancer proliferation via the $W n t / \beta$-catenin pathway [3] and JAK/STAT3 signaling pathway [4], respectively. Recently, we found that RNF6 is highly expressed in leukemia and promotes leukemogenesis, thereby conferring resistance to doxorubicin, a major antileukemia agent [5]. Furthermore, total saponins from Paris forrestii displayed potent antileukemia activity when used to target RNF6 [6]. Overall, RNF6 plays a key role in various cancers, but its specific functions in multiple myeloma $(\mathrm{MM})$ remain elusive.

$\mathrm{MM}$ is a class of hematological malignancies derived from clonal plasma cells. Among the approved drugs for MM therapy, glucocorticoids, such as dexamethasone and prednisone, are commonly prescribed. Glucocorticoids exert anti-MM activity by activating glucocorticoid receptor (GR), one of the major nuclear transcription factors. When GR is activated, it is translocated to the nucleus, where it binds to the GR-responsive element (GRE) on the promoter regions of target genes associated with cell apoptosis, thereby promoting their transcription. Unfortunately, resistance to dexamethasone has been frequently observed in MM patients, but the mechanism for this resistance is largely unknown. Both decreased expressions of GR and the reduced or erratic binding activity of GR were reported to confer resistance to dexamethasone $[7,8]$. Moreover, as it is a major transcription factor with diverse functions, GR has been demonstrated to modulate more than 900 genes in MM by global gene expression analyses; these genes include not only proapoptotic genes but also prosurvival genes, such as $\mathrm{Bcl}-\mathrm{xL}$ and $\mathrm{Mcl}-1$ [9]. In addition, GR transcriptional activities are also modulated by various posttranslational modifications (PTMs) including acetylation, phosphorylation, sumoylation, and ubiquitination [10-12]. For example, the E3 ligase CHIP can mediate GR turnover via the ubiquitin-proteasome pathway, thereby suppressing its transcriptional activity [13].

In the present study, we found that the ubiquitin ligase RNF6 is highly expressed in MM cells. RNF6 can induce K63-linked

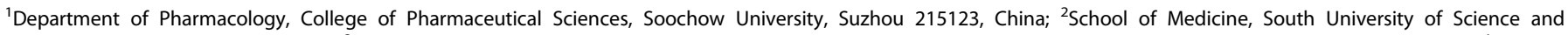

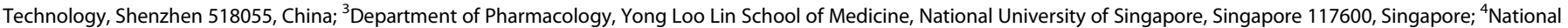

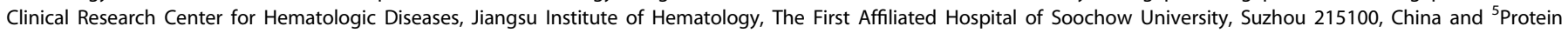

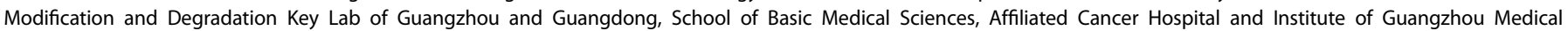
University, Guangzhou 511436, China

Correspondence: Xiao-wen Tang (xwtang1020@163.com) or Xin-liang Mao (xinliangmao@gzhmu.edu.cn)
}

Received: 13 June 2019 Accepted: 12 September 2019

Published online: 23 October 2019 
polyubiquitination of GR and increase its stability. As a result, RNF6 upregulates GR transcriptional activity and promotes MM cell survival by inducing the expression of prosurvival genes. Thus, targeting the RNF6/GR axis may be a novel strategy for MM treatment.

\section{MATERIALS AND METHODS}

Cells and cell culture

MM cell lines (KMS11, RPMI-8226, OPM2, LP1, MM1.S, MM1.R, OClMY5, and U266 cells) and a leukemia cell line (K562 cells) were purchased from American Type Culture Collection (Manassas, Virginia, USA) or generously provided by Dr Aaron D. Schimmer, Princess Margaret Cancer Center, Toronto, ON, Canada. HEK293T and HeLa cells were provided by $\mathrm{Dr}$ Michael F. Moran, The University of Toronto, Toronto, ON, Canada. Cell culture followed protocols described previously [5].

Primary bone marrow aspirates from MM patients and volunteer healthy donors were collected from the Department of Hematology, The First Affiliated Hospital of Soochow University. The primary aspirates were used to isolate mononuclear cells by Lympholyte ${ }^{\circledR}$ cell separation media (Cedarlane, Canada) according to the manufacturer's instructions as described previously [14]. The collection and use of human tissues for this study was approved by the Institutional Review Board of Soochow University. Informed consent was obtained in accordance with the Declaration of Helsinki.

Reverse transcription-polymerase chain reaction (RT-PCR) Total RNA was extracted from cells of interest using TRIzol ${ }^{\circledR}$ reagent according to the manufacturer's instructions (Thermo Fisher Scientific, Shanghai, China). First-strand cDNA was generated from equal quantities of total RNA using EasyScript ${ }^{\circledR}$ First-Strand cDNA Synthesis SuperMix ${ }^{\otimes}$ (TransGen Biotech Co., Ltd., Beijing, China). PCR was performed in a $25 \mu \mathrm{L}$ reaction system containing $12.5 \mu \mathrm{L}$ of $2 \times$ Easy Taq SuperMix (TransGen), $1 \mu \mathrm{L}$ of $\mathrm{cDNA}, 0.5 \mu \mathrm{L}$ of each primer, and $10.5 \mu \mathrm{L}$ of sterile, pure $\mathrm{H}_{2} \mathrm{O}$. The primers used were as follows: RNF6, forward 5'CATCAGTGGCTCTTCGGTCA-3' and reverse $5^{\prime}$-ATGCTCATAGT GCCTGGTGG-3'; GAPDH, forward 5'-AGTCCACTGGCGTCTTCA-3' and reverse $5^{\prime}$-CTCCGACGCCTGCTTCACCA-3'. Reaction cycling conditions were $3 \mathrm{~min}$ at $95^{\circ} \mathrm{C}$, followed by 30 cycles at $95^{\circ} \mathrm{C}$ for $30 \mathrm{~s}, 60^{\circ} \mathrm{C}$ for $30 \mathrm{~s}$, and $72{ }^{\circ} \mathrm{C}$ for $40 \mathrm{~s}$, and $1 \mathrm{cycle}$ at $72{ }^{\circ} \mathrm{C}$ for $10 \mathrm{~min}$. Products were analyzed on $2 \%$ agarose gels (TransGen).

\section{Quantitative real-time PCR (qRT-PCR)}

Total RNA was prepared as described above, followed by cDNA synthesis using EasyScript First-Strand CDNA Synthesis SuperMix (TransGen). qRT-PCR was performed using Luminaris Color HiGreen High ROX qPCR Master Mix (Thermo Fisher Scientific) with a Step One Plus ${ }^{T M}$ real-time PCR system (Thermo Fisher Scientific). The primers used for qRT-PCR were listed in Table 1.

Preparation of lentiviral RNF6 and shRNF6

The full-length RNF6 gene was amplified by PCR with the primers 5'-CCCGGAATTCATGAATCAGTCTAGATCGAGATCAG-3' (forward) and 5'-AAATATGCGGCCGCTTACCCATTGTTGCTATGTTAGACCC-3' (reverse) flanked with EcoRI and Notl recognition sites, respectively. To generate lentiviral RNF6, RNF6 was inserted between $E c o R I$ and BamHI recognition sites in the pLVX-AcGFP lentiviral vector (Thermo Fisher Scientific). Lentiviral particles were produced by a specific protocol described previously [15]. Lentivirusdelivered shRNA against RNF6 (shRNF6) and negative control shRNA (shNC) were purchased from Gene Chemical Technology Co., Ltd. (Shanghai, China). The shRNF6 target sequence was $5^{\prime}$ ATAACAGTTCCTCTTCGTA-3'.

\begin{tabular}{|lll|}
\hline Table 1. & The primers for qRT-PCR & \\
\hline Gene Name & Primer name & Primers' sequences $\left(5^{\prime}-3^{\prime}\right)$ \\
\hline BCL2L1 & Forward & GAGCTGGTGGTTGACTTTCTC \\
& Reverse & TCCATCTCCGATTCAGTCCCT \\
RRM2 & Forward & CACGGAGCCGAAACTAAAGC \\
& Reverse & TCTGCCTTCTTATACATCTGCCA \\
SGK1 & Forward & CCCCCTTTTAACCCAAATGT \\
& Reverse & CAGGCTCTTCGGTAAACTCG \\
Mcl-1 & Forward & TGCTTCGGAAACTGGACATC \\
& Reverse & TAGCCACAAAGGCACCAAAAG \\
RNF6 & Forward & AGAAGATGGCAGCAAGAGCG \\
& Reverse & TCAAGTCAGGCTGAGATGCTAGT \\
GAPDH & Forward & GCACCGTCAAGGCTGAGAAC \\
& Reverse & TGGTGAAGACGCCAGTGGA \\
\hline
\end{tabular}

Cell viability

MM cells at an initial density of $2 \times 10^{4}$ cells/well in 96-well plates were transfected with siRNF6 or infected with pLVX-RNF6 or scramble lentivirus and cultured for the indicated periods. Cell viability was determined by the MTT assay as described previously [6].

Immunoblotting (IB)

Whole-cell lysates were prepared as described previously [14]. Equal amounts $(30 \mu \mathrm{g})$ of total proteins were subjected to sodium dodecyl sulfate polyacrylamide gel electrophoresis (SDS-PAGE) separation, followed by IB assays with specific polyclonal antibodies against RNF6, GRa (GR), TLE3, SHP-1, ER, AR, GAPDH, a-tubulin (Proteintech, Rosemont, IL, USA), Ub (Santa Cruz Biotechnology, Inc., Santa Cruz, CA, USA), Flag, Myc, HA (MBL Hangzhou Biotech Co., Ltd., Hangzhou, Zhejiang, China), K63-Ub, $\mathrm{BCl}-\mathrm{xL}$, and $\mathrm{Mcl}-1$ (Cell Signaling Technology Co., Boston, MA, USA). Anti-mouse and anti-rabbit lgG horseradish peroxidaseconjugated antibodies were purchased from Beyotime Institute of Biotechnology (Nantong, Jiangsu, China).

\section{Construction of truncated GR variants}

GR coding sequences were obtained by the NCBI website (http:// www.ncbi.nlm.nih.gov), and a series of truncated $G R$ variants were amplified by PCR using the primers listed in Table 2, followed by insertion into the pcDNA3.1 vector with a Flag tag (Invitrogen).

Small interfering RNA (siRNA) transfection

siRNAs against RNF6 or GR and a control siRNA were synthesized by GenePharma (Shanghai, China). The specific sequences targeting RNF6 (siRNF6) were as follows: siRNF6\#1: 5'- GUACAGCA AACCACUAGAATT-3' (sense) and 5'-UUCUAGUGGUUUGCUGUA CTT-3' (antisense); and siRNF6\#2: 5'-GGCUAGAAGUAAUGUUAC ATT-3' (sense) and 5'-UGUAACAUUACUUCUAGCCTT-3' (antisense). The specific sequences targeting GR (siGR) were as follows: $5^{\prime}$ GAUGUAAGCUCUCCUCCAUTT-3' (sense) and 5'-AUGGAGGAGA GCUUACAUCTT-3' (antisense). The negative control siRNA (siNC) sequences were as follows: 5'-UUCUCCGAACGUGUCACGUTT-3' (sense) and 5'-ACGUGACACGUUCGGAGAATT-3' (antisense). siRNAs were transfected into RPMI-8226 and LP1 cells using a RiboFECT CP transfection kit (RiboBio Biotech, Inc., Guangzhou, China) according to the manufacturer's instructions.

Luciferase assay

A GRE-driven luciferase reporter plasmid (pGRE.Luci) was purchased from Beyotime. pGRE.Luci and associated plasmids were transfected into HEK293T cells for $36 \mathrm{~h}$ by polyethylenimine (PEI, Sigma-Aldrich, St. Louis, MO, USA) as reported previously [16]. Cell 


\begin{tabular}{|lll|}
\hline Table 2. & The primers for constructs of GR truncates \\
\hline GR (AA) & Primer name & Primers' sequences $\left(5^{\prime}-3^{\prime}\right)$ \\
\hline WT & Forward & CGGGATCCATGGACTCCAAAGAATCATTAACTC \\
& Reverse & CCGCTCGAGTCACTTTTGATGAAACAGAAGTT \\
$402-777$ & Forward & CGGGATCCATGGTAAGCTCTCCTCCATCCAG \\
& Reverse & CCGCTCGAGTCACTTTTGATGAAACAG \\
$1-494$ & Forward & CGGGATCCATGGACTCCAAAGAATC \\
& Reverse & CCGCTCGAGTCACTTTGTTTTCGAGCTTC \\
$1-530$ & Forward & CGGGATCCATGGACTCCAAAGAATC \\
& Reverse & CCGCTCGAGTCAAGGGGTGAGTTGTGGTAA \\
$531-777$ & Forward & CGGGATCCATG ACCCTGGTGTCACTGTTG \\
& Reverse & CCGCTCGAGTCACTTTTGATGAAACAG
\end{tabular}

lysates were subjected to luciferase analysis using the Bright-Glo system (Promega ${ }^{\circledR}$, Madison, WI, USA) as described previously [16]. Luciferase activity was normalized to $\beta$-gal expression for each sample. All transfection experiments were performed in triplicate.

Cycloheximide (CHX) chase assay

After transfection with plasmids of interest for $24 \mathrm{~h}$, HEK293T cells were treated with $\mathrm{CHX}(100 \mu \mathrm{g} / \mathrm{mL}$, Sigma-Aldrich) for certain time periods as needed. Cell lysates were then prepared for SDS-PAGE and IB analyses with specific antibodies.

Co-immunoprecipitation (ColP)

HEK293T cells were transfected with specific plasmids as needed by PEl. Thirty-six hours later, cell lysates were prepared for colP as described previously [5]. First, cell lysates were incubated with an anti-specific antibody overnight at $4{ }^{\circ} \mathrm{C}$, followed by incubation with protein $A+G$ Sepharose beads (Beyotime) for $4 \mathrm{~h}$. The coprecipitated proteins were subjected to IB assays against specific antibodies.

\section{Flow cytometric analysis}

To assess cell apoptosis, MM cells were infected with lentiviral shRNF6 for $96 \mathrm{~h}$, followed by Annexin $\mathrm{V}$ and propidium iodide staining (Beyotime) and flow cytometric analysis. The specific protocol used was described previously [16].

Statistical analysis

Student's $t$ test was used for comparisons between two groups in the study. All statistical tests were two-sided, and a $P$ value $<0.05$ indicated statistical significance.

\section{RESULTS}

RNF6 is overexpressed in MM cells and promotes MM cell proliferation

Our previous studies showed that RNF6 is highly expressed in leukemia but not normal blood cells [5]. To determine the role of RNF6 in MM, we first measured the expression profile of RNF6 in MM cell lines by using the K562 typical leukemia cell line as a positive control. As shown in Fig. 1a, RNF6 was highly expressed in all MM cell lines tested as well as K562 cells. RNF6 was also highly expressed in embryonic kidney cells (HEK293T) and cervical carcinoma cells (HeLa). To investigate whether RNF6 was also elevated in MM patients, primary bone marrow cells from MM patients and healthy donors were collected, and RNF6 mRNA levels were evaluated by qRT-PCR. As shown in Fig. 1b, the mRNA level of RNF6 in MM patients was significantly higher than that in normal bone marrow cells. This finding was further confirmed by RT-PCR, as shown in Fig. 1c. As MM gradually evolves from MGUS and smoldering MM (SMM), we next analyzed the public database
Oncomine (https://www.oncomine.org) and found that RNF6 is expressed in SMM, as shown in Fig. 1d, although no data from MM patients were available. Because RNF6 was highly expressed in MM cell lines and MM patient samples but not normal blood cells, we wondered whether RNF6 can promote MM cell proliferation. To this end, RNF6 was knocked down by siRNA in MM cell lines (LP1 and RPMI-8226), and an MTT assay was performed. The knockdown of RNF6 significantly decreased MM cell proliferation (Fig. 1e, f). In contrast, overexpression of RNF6 promoted the proliferation of MM cells (Fig. 1g, h). To determine whether RNF6 promotes MM cell survival, RNF6 was knocked down in MM cells, followed by an apoptosis assay. As shown in Fig. 1i, when RNF6 was knocked down, the proportion of Annexin V-positive cells was drastically increased in both the LP1 and RPMI-8226 cell lines, suggesting that RNF6 promotes MM cell survival. These results thus indicated that RNF6 is highly expressed in MM cells and promotes MM cell proliferation and survival.

RNF6 mediates K63-linked polyubiquitination of GR

As it is a RING-type ubiquitin ligase, RNF6 has been found to mediate the ubiquitination of several proteins including the nuclear protein AR and ER [2, 17]. GR is an important nuclear protein in MM cells. Therefore, we next determined whether GR can be ubiquitinated by RNF6. To this end, Flag-tagged GR and Myc-tagged RNF6 were cotransfected into HEK293T cells, followed by IP/IB assays. The levels of polyubiquitinated GR were markedly increased in cells transfected with Myc-RNF6 (Fig. 2a). However, when the essential RING domain was deleted (Myc- $\Delta$ RING), RNF6 lost its ability to mediate GR ubiquitination (Fig. 2a). This experiment suggested that RNF6 is a ubiquitin ligase of GR. To confirm this hypothesis, RNF6 was knocked down by specific interfering RNA, followed by an evaluation of GR ubiquitination. GR ubiquitination was dramatically reduced by two different siRNAs (Fig. 2b). To determine whether RNF6 has a similar effect on GR in MM cells, the LP1 and RPMI-8226 MM cell lines were infected with lentiviral RNF6, followed by ubiquitination analyses. Consistent with the observations shown in Fig. 2a, b, RNF6 significantly increased GR polyubiquitination levels in both MM cell lines (Fig. 2c).

Protein ubiquitination involves the attachment of various ubiquitin chain types. RNF6 has been reported to induce $\mathrm{K} 6 /$ $\mathrm{K} 27-$ and K48-linked polyubiquitination $[2,18]$. To determine the specific type of ubiquitin chain linkage to GR mediated by RNF6, we screened a series of mutant ubiquitin plasmids with only one intact lysine residue. Among the seven ubiquitin plasmids, GR was mainly ubiquitinated in the presence of Ub-Lys48 and Ub-Lys63 (Fig. 2d), suggesting that GR undergoes both K48- and K63-linked polyubiquitination. We next evaluated GR ubiquitination levels in the presence of RNF6. As shown in Fig. 2e, RNF6 mainly mediated the K63-linked, but not K48-linked, ubiquitination on GR. Notably, Myc-RNF6 $\Delta$ RING failed to markedly increase GR ubiquitination. To further confirm this finding, we knocked down RNF6 by specific shRNA to examine endogenous levels of K63-linked ubiquitinated GR in MM cell lines. Consistent with the above findings, when RNF6 was knocked down, the K63-linked ubiquitination of GR was abolished in MM cell lines (Fig. 2f). These findings thus suggest that RNF6 induces K63-linked GR polyubiquitination.

RNF6 increases the stability of GR

RNF6 has been reported to induce the degradation of SHP-1 and $\operatorname{TLE} 3[3,4]$ but increase the steroid receptors AR and ER $[2,17]$. The above results showed that RNF6 mediates GR polyubiquitination; to determine the effects of RNF6 on GR stability, wild-type or RING domain deletion $(\triangle \mathrm{RING})$ plasmids were cotransfected with a $G R$ plasmid, followed by IB assay. As shown in Fig. 3a, RNF6 markedly increased GR protein levels in HEK293T cells in a concentrationdependent manner. To our surprise, RNF6 $\Delta$ RING also increased GR stability in a manner similar to that of WT RNF6 (Fig. 3a). To 
a 293T HeLa KMS OPM2 8226 LP1 1.S 1.R U266 MY5 K562 RNF6 a-tubulin

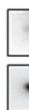

C
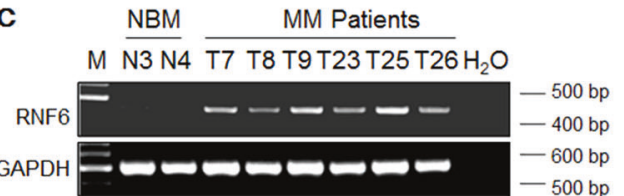

b

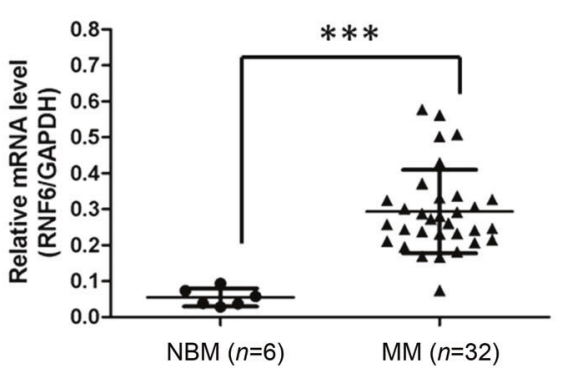

e

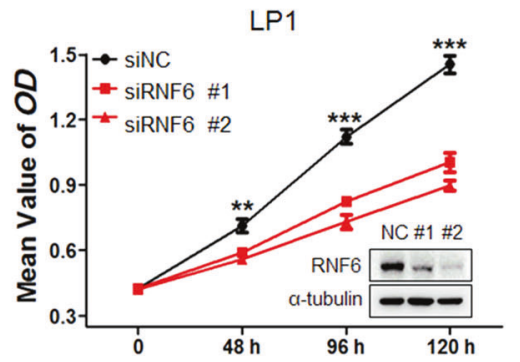

g

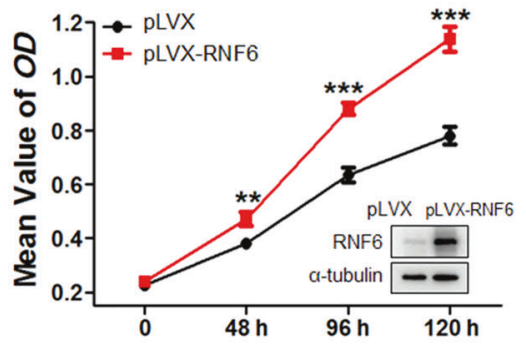

i

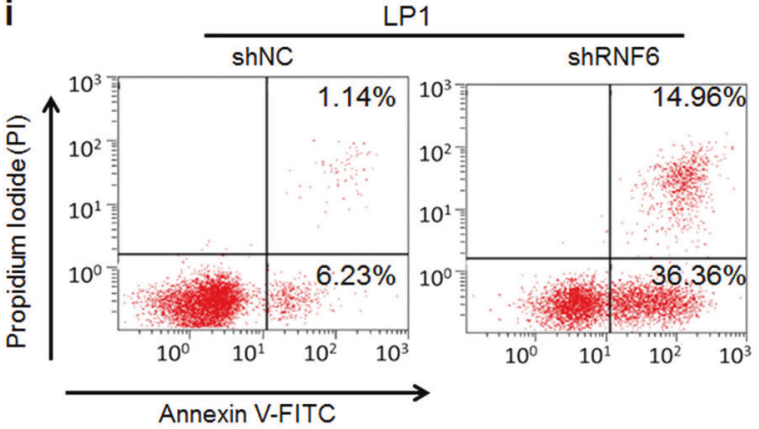

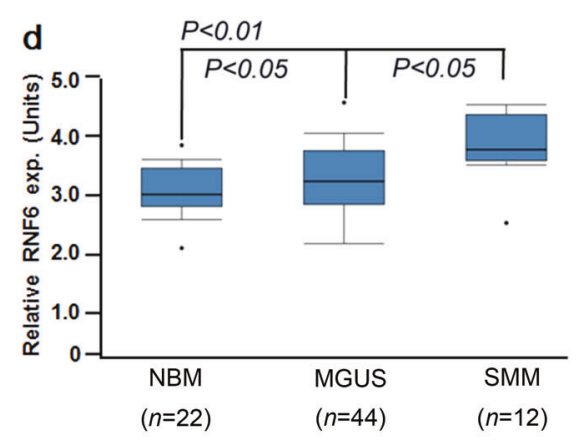

f

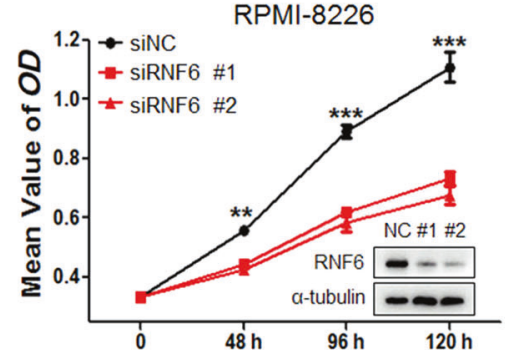

h

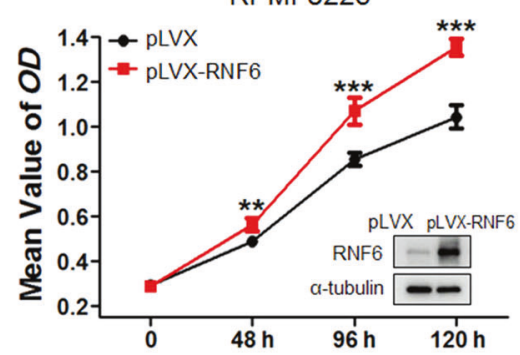

Fig. 1 RNF6 is overexpressed in MM cells and promotes MM cell proliferation. a Cell lysates from various cell lines were subjected to IB against RNF6. b Bone marrow cells from $32 \mathrm{MM}$ patients and six healthy donors were subjected to qRT-PCR analysis. c Representative MM patients and normal controls were subjected to RT-PCR to measure RNF6 mRNA levels. $\mathbf{d}$ The RNF6 expression level was analyzed based on the Oncomine database (https://www.oncomine.org). RNF6 mRNA expression was analyzed from healthy donors $(n=22)$, MGUS patients $(n=44)$, and SMM patients $(n=12)$ based on the Zhan myeloma 3 dataset. LP1 cells $(\mathbf{e})$ and RPMI-8226 cells (f) were transfected with siRNF6 or siNC, followed by cell counting using the MTT assay at the indicated times. LP1 cells (g) and RPMI-8226 cells (h) were infected with lentiviral RNF6 (pLVX-RNF6), followed by cell counting using the MTT assay at the indicated times. i RNF6 was knocked down in LP1 and RPMI-8226 cells with specific short hairpin RNA (shRNA) for $96 \mathrm{~h}$, followed by Annexin V/PI staining and flow cytometric analysis. ${ }^{* *} P<0.01,{ }^{* * *} P<0.001$. KMS: KMS11; 8226: RPMI-8226; 1.S: MM1.S; 1.R: MM1.R; MY5: OCI-MY5 


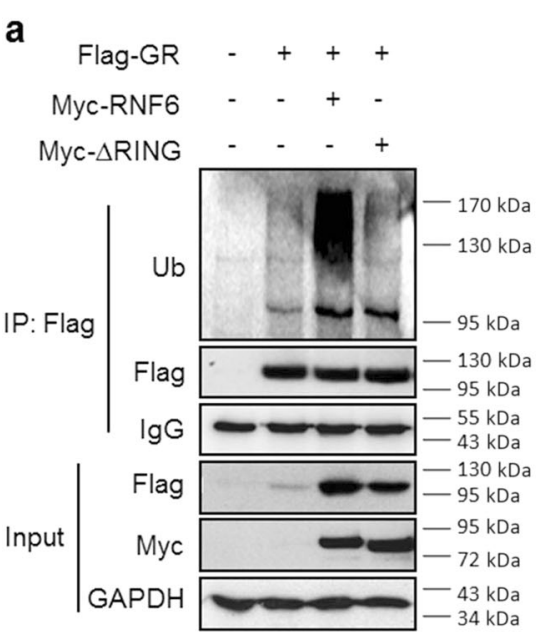

C

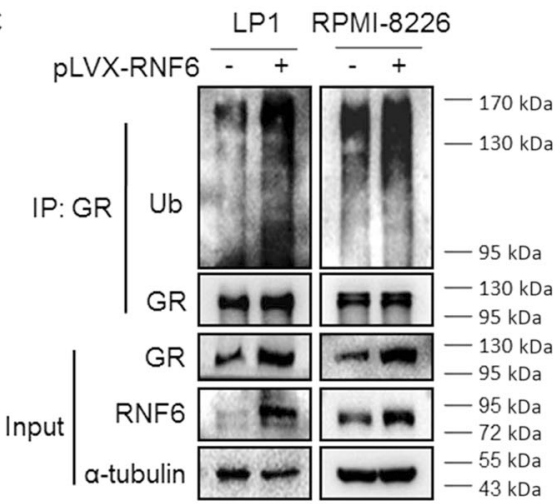

b

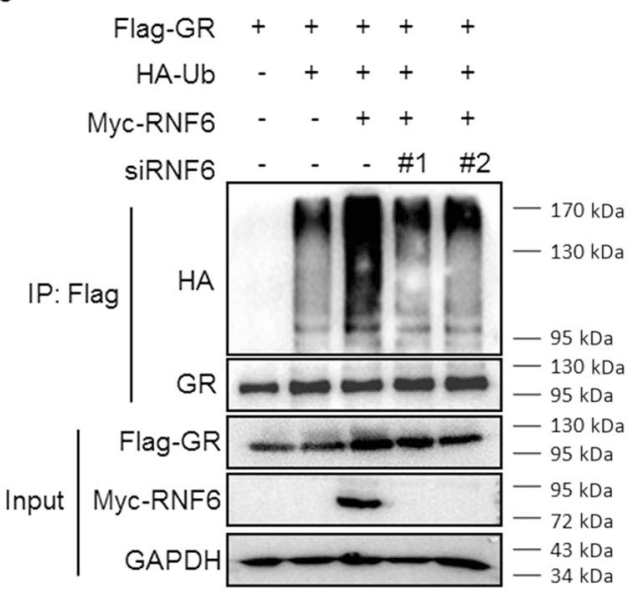

d

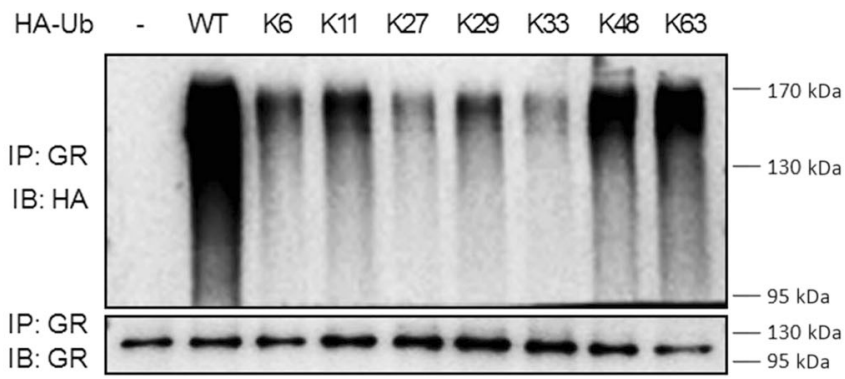

f

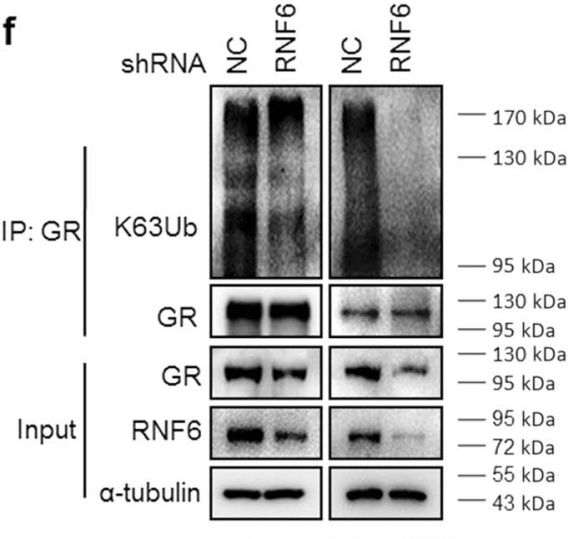

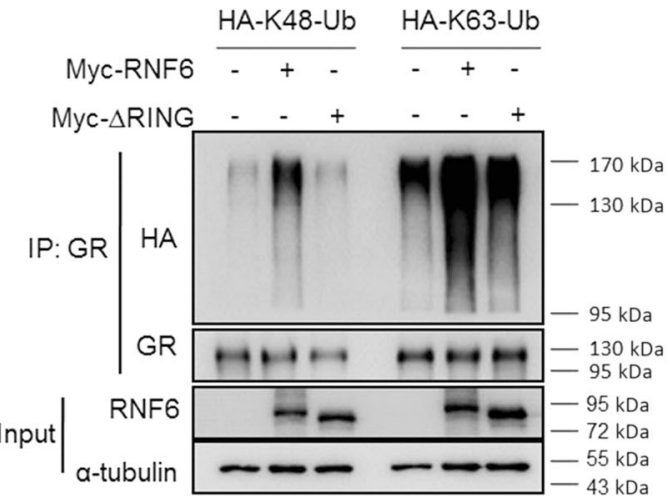

LP1 RPMI-8226

Fig. 2 RNF6 mediates the K63-linked polyubiquitination of GR. a HEK293T cells were cotransfected with Flag-GR and Myc-RNF6 or MycRNF6 2 RING plasmids for $36 \mathrm{~h}$, followed by IP/IB assays as indicated. b HEK293T cells were cotransfected with Flag-GR, HA-Ub, and Myc-RNF6 with or without siRNF6, followed by IP/IB assays as indicated. c LP1 and RPMI-8226 cells were infected with lentiviral RNF6 for $72 \mathrm{~h}$, followed by IP/IB assays as indicated. d HEK293T cells were transfected with HA-tagged wild-type (WT) ubiquitin or a single lysine-containing mutant as indicated, followed by IP/IB assays. e HEK293T cells were transfected with HA-K48 or HA-K63 ubiquitin plasmids with RNF6 or RNF6 2 RING. Thirty-six hours later, cell lysates were subjected to IP/IB assays as indicated. $\mathbf{f}$ LP1 and RPMI-8226 cells were infected with RNF6 shRNA, and $96 \mathrm{~h}$ later, cell lysates were prepared for IP/IB assays to evaluate endogenous K63-linked ubiquitinated GR

determine the regulatory effect of RNF6 on endogenous GR, we chose three cell lines that express GR: HeLa, LP1, and RPMI-8226 cells. As shown in Fig. 3b, c, RNF6 dramatically increased endogenous GR in HeLa cells at the protein level but did not alter the GR mRNA expression level, suggesting that RNF6 stabilizes endogenous GR. Notably, this correlation between RNF6 and GR was statistically significant in additional MM cell lines, as shown in Fig. 3d, e. In these assays, the ectopic expression of RNF6 markedly increased GR (Fig. 3d). Previous studies have demonstrated that the proteins AR, ER, SHP-1, and TLE3 are substrates of RNF6 in various cancers; to determine whether RNF6 specifically modulates GR in MM cells, RNF6 was knocked down in the LP1 and RPMI-8226 MM cell lines, followed by IB assays. All of the tested proteins except for AR were detectable in MM cells (Fig. 3e). However, knockdown of RNF6 did not markedly alter the protein levels of SHP-1 and TLE3, and the ER protein level 
a

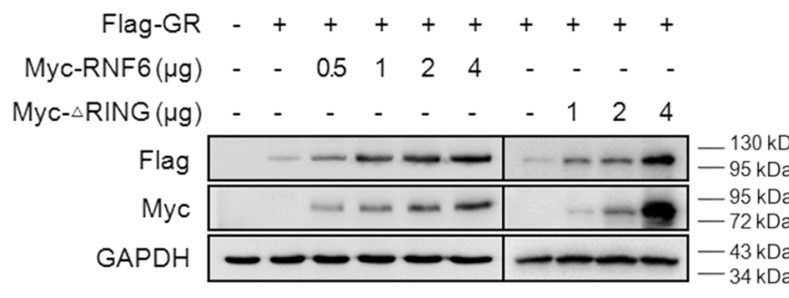

b

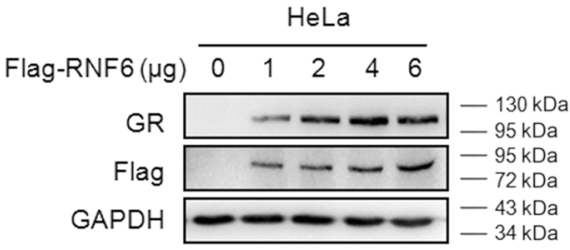

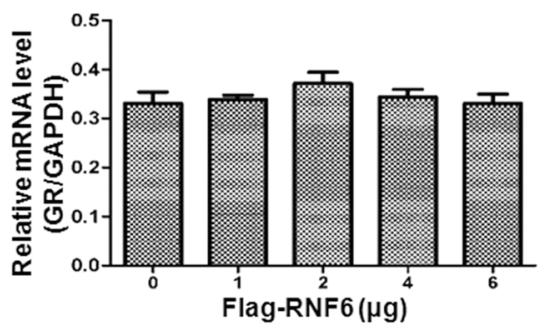

e

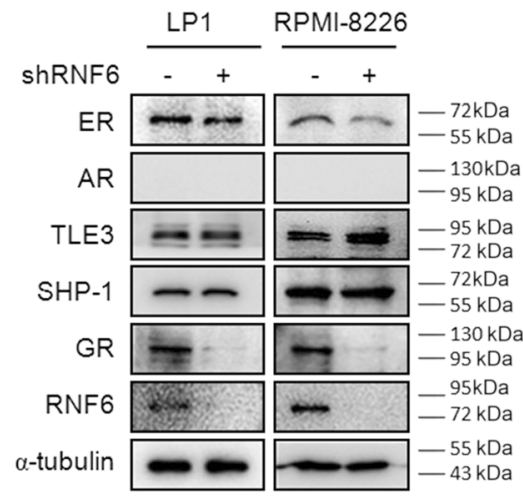

d

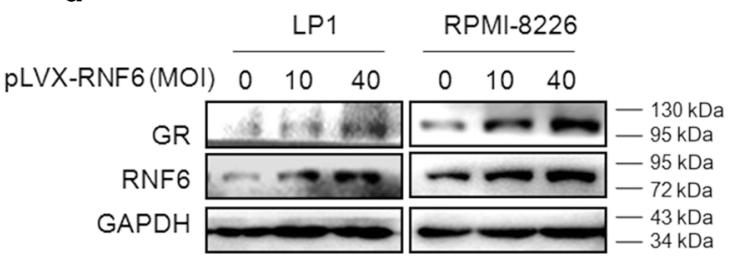

$\mathbf{f}$

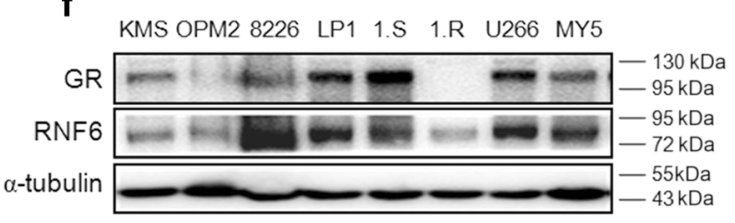

g

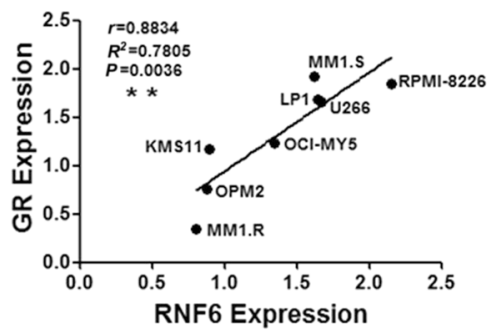

h
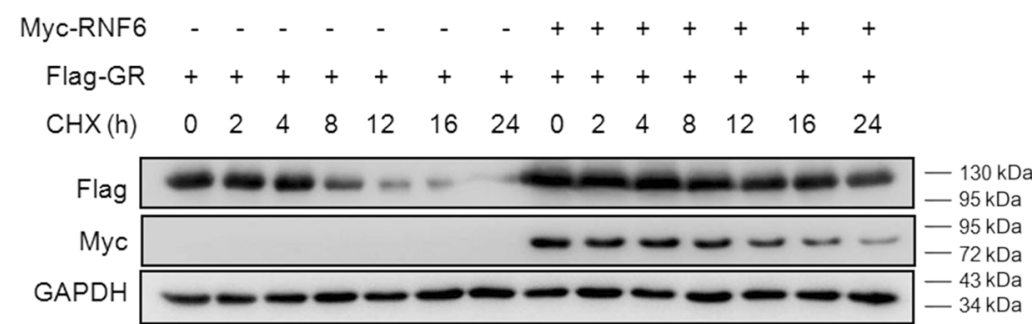

Fig. 3 RNF6 increases the stability of the GR protein. a Flag-GR plasmids were cotransfected with increased Myc-RNF6 or Myc-RNF6 $\triangle$ RING (Myc- $\Delta$ RING) for $36 \mathrm{~h}$, followed by IB assays as indicated. RNF6 was transfected into HeLa cells at increasing concentrations for $36 \mathrm{~h}$, followed by IB (b) or RT-PCR (c) to measure the protein and mRNA levels of GR, respectively. d LP1 and RPMI-8226 cells were infected with increasing amounts of lentiviral RNF6 for $72 \mathrm{~h}$, followed by IB assays. e LP1 and RPMI-8226 cells were infected with shRNF6 for $96 \mathrm{~h}$, followed by IB assays against specific antibodies as indicated. $\mathbf{f}$ Cell lysates from Fig. 1a were subjected to IB assays against GR. $\mathbf{g}$ Correlation analysis of RNF6 and GR expression in MM cell lines from $\mathbf{f}$ and Fig. 1a after normalization to $\alpha$-tubulin expression was performed. $\mathbf{h}$ RNF6 and GR plasmids were cotransfected into HEK293T cells for $24 \mathrm{~h}$, followed by CHX $(100 \mu \mathrm{g} / \mathrm{mL})$ treatment for the indicated time periods and IB assays

was slightly decreased. In contrast, GR was significantly decreased following RNF6 knockdown (Fig. 3e). This assay indicated that RNF6 probably specifically modulates GR in MM cells. We next assessed the correlation between RNF6 and GR expression in a panel of MM cell lines. As shown in Fig. 3f, $g$, the protein levels of GR were significantly correlated with the expression of RNF6. To further evaluate the regulatory effect of RNF6 on GR protein stability, a CHX chase assay was performed. As shown in Fig. 3h, when GR synthesis was inhibited by CHX, the addition of RNF6 prevented the degradation of GR, and the half-life of GR was increased from 8 to $24 \mathrm{~h}$. Therefore, these results collectively suggest that RNF6 increases the stability of GR.
RNF6 interacts with GR at the ligand-binding domain (LBD)

Next, we determined whether RNF6 interacts with GR. To this end, a reciprocal co-IP assay was performed in HEK293T cells after transfection of both the RNF6 and GR plasmids. As shown in Fig. 4a, RNF6 was found in the GR immunoprecipitates, and GR was found in the RNF6 immunoprecipitates, suggesting that RNF6 and GR interact in HEK293T cells. Moreover, this interaction was also found in $M M$ cells (Fig. 4b). Notably, RNF6 $\triangle$ RING also interacted with GR (Fig. 4a), suggesting that the RING domain is disposable for the interaction of RNF6 and GR. Therefore, both ectopic and endogenous RNF6 can interact with GR. To identify the specific region in GR responsible for its interaction with RNF6, 
a

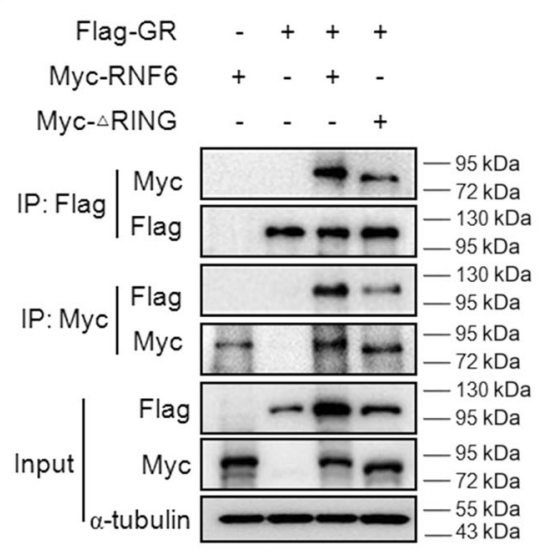

b

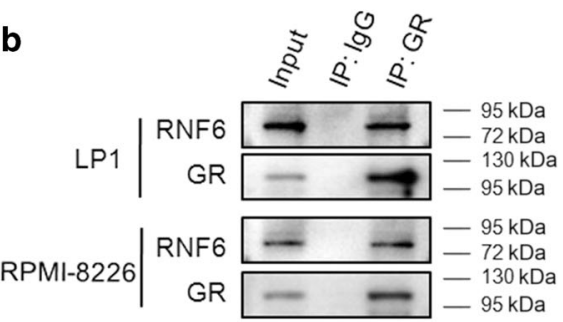

C

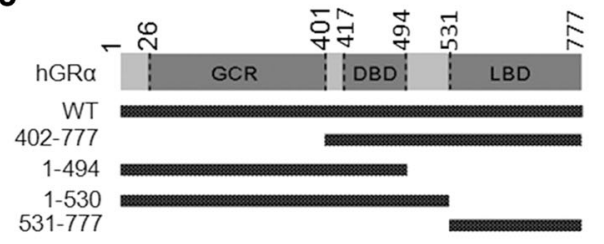

d Myc-RNF6 - + + + + +

Flag-GR-WT - + - - - -

$1-494-\quad+-\quad-$

$1-530-\quad-++-$

402-777 - - - - + -

$531-777-\quad-\quad-\quad+$

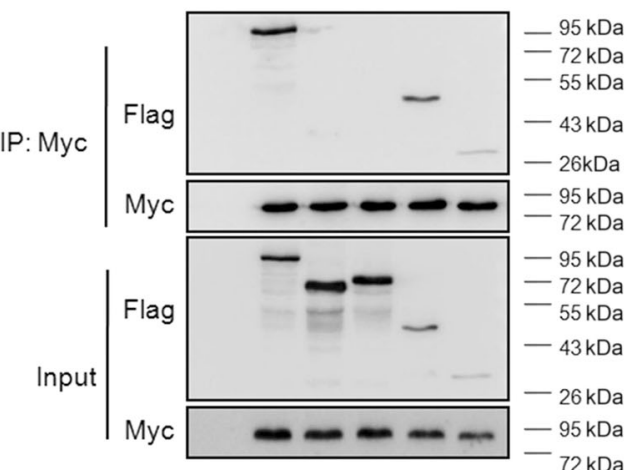

Fig. 4 RNF6 interacts with GR at the ligand-binding domain. a HEK293T cells were cotransfected with Flag-GR and Myc-RNF6 or MycRNF6 $\Delta$ RING (Myc- $\Delta$ RING) plasmids. After $36 \mathrm{~h}$, cells were harvested and subjected to reciprocal IP/IB assays. b Total LP1 and RPMI-8226 cell lysates were extracted for IP/IB assays against GR antibodies as indicated. c A schematic of truncated GR proteins. d Truncated GR protein plasmids were transfected into HEK293T cells along with Myc-RNF6. Thirty-six hours later, the cells were lysed and subjected to IP/IB assays

a series of GR proteins truncated according to its structural domains (its N-terminal domain, DNA-binding domain (DBD), and LBD) were generated (Fig. 4c) [19]. After the cotransfection of GR variants with RNF6 in HEK293T cells, co-IP/IB assays were performed. RNF6 was found in fragments consisting of amino acids 402-777 (containing the LBD and DBD) and 531-777 (containing the LBD) but not in fragments consisting of amino acids 1-494 and 1-530 (containing the DBD). Therefore, we believe that the LBD, but not the DBD, of GR is essential for the interaction between GR and RNF6 (Fig. 4d). These results thus suggest that RNF6 can bind to GR at the LBD.

RNF6 enhances GR activity and promotes the expression of prosurvival genes in MM cells

GR, which belongs to the nuclear receptor superfamily, is a transcription factor essential for modulating a broad, cell typespecific spectrum of genes [20]. The above investigation revealed that RNF6 not only increases GR stability but also binds to its LBD, suggesting that RNF6 promotes GR transcriptional activity. To this end, we constructed a pGRE-luci reporter plasmid (GR responsive element-driven luciferase reporter) and cotransfected it in HEK293T cells with GR, RNF6 or both, followed by the luciferase activity assay. RNF6 dramatically promoted pGRE-luci activity (Fig. 5a). In contrast, knockdown of RNF6 significantly decreased pGRE-luci activity (Fig. 5b).

To directly analyze the effect of RNF6 on GR-induced gene expression, the mRNA levels of four genes, BCL2L1, RRM2, SGK1, and Mcl-1, were determined. As shown in Fig. 5c, knockdown of RNF6 obviously downregulated the mRNA levels of all four genes in both LP1 and RPMI-8226 cells. These findings were confirmed by IB assays in MM cells after the knockdown or forced expression of RNF6. In accordance with the qRT-PCR results, expression levels of the $\mathrm{BCl}-\mathrm{xL}$ (the protein encoded by $\mathrm{BCL} 2 \mathrm{~L} 1$ ) and $\mathrm{Mcl}-1$ proteins were markedly induced by RNF6 in MM cells after the ectopic expression of RNF6 (Fig. 5d). Consistently, when RNF6 was knocked down in MM cells, $\mathrm{BCl}-\mathrm{xL}$ and $\mathrm{Mcl}-1$ levels were decreased accordingly (Fig. 5e). To determine whether RNF6 promotes $\mathrm{BCl}-\mathrm{xL}$ and $\mathrm{Mcl}-1$ expression via GR, GR was knocked down by siRNA, followed by lentiviral RNF6 infection and IB assays. When GR was intact, RNF6 dramatically promoted the expression of $\mathrm{BCl}-\mathrm{xL}$ and $\mathrm{Mcl}-1$, but RNF6 failed to promote the expression of Bcl-xL and Mcl-1 when GR was knocked down (Fig. 5f), suggesting that GR is essential for RNF6 to promote $\mathrm{BCl}-\mathrm{xL}$ and $\mathrm{Mcl}-1$ expression. All the above results indicated that RNF6 enhances GR activity and promotes cell survival in MM.

Overexpression of RNF6 reduces the anti-MM activity of dexamethasone

Dexamethasone is a potent anti-MM steroid that acts via GR signaling, and the above results showed that RNF6 stabilizes GR and promotes the expression of prosurvival genes in MM. Therefore, RNF6 probably antagonizes the anti-MM activity of dexamethasone. To verify this hypothesis, both LP1 and RPMI-8226 cells were infected with lentiviral RNF6, followed by dexamethasone treatment. Cell viability was thereafter determined by MTT assay. The ectopic expression of RNF6 significantly increased MM cell viability; in contrast, dexamethasone dramatically decreased MM cell viability. Notably, the addition of RNF6 partly but markedly abolished dexamethasone-decreased cell proliferation (Fig. 6a, b). These results suggest that overexpression of RNF6 can antagonize the action of dexamethasone in MM, which is probably the mechanism of dexamethasone resistance in MM.

\section{DISCUSSION}

In the present study, we demonstrate that RNF6 is highly expressed in MM, promotes MM cell proliferation and survival, 
a

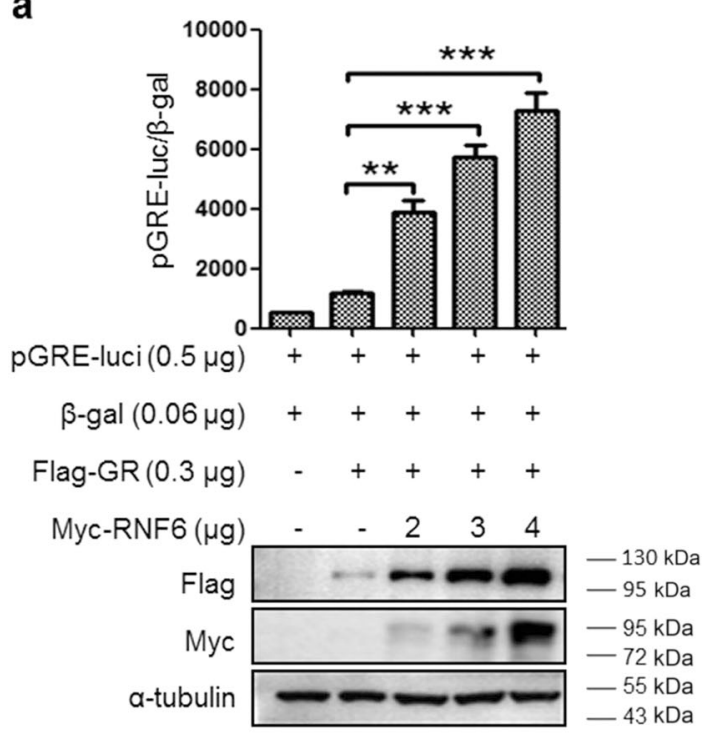

C

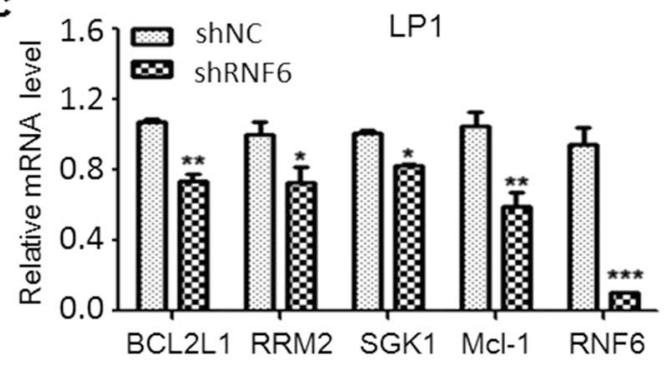

d

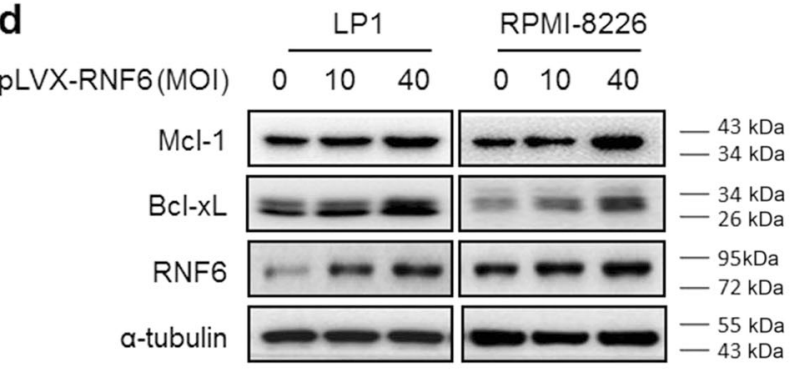

f

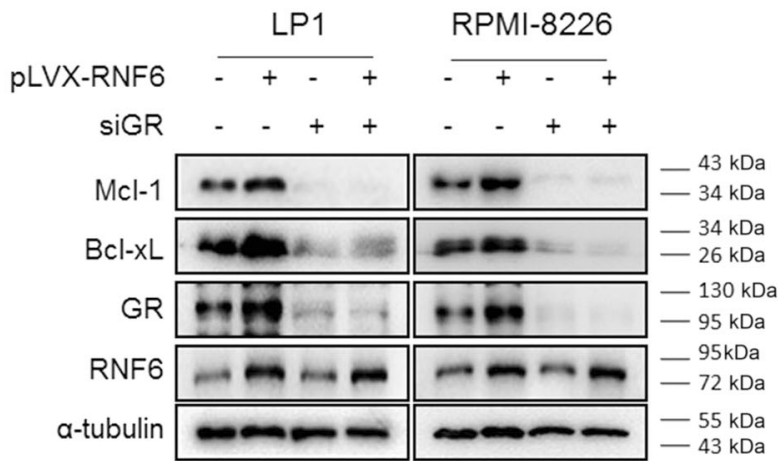

b

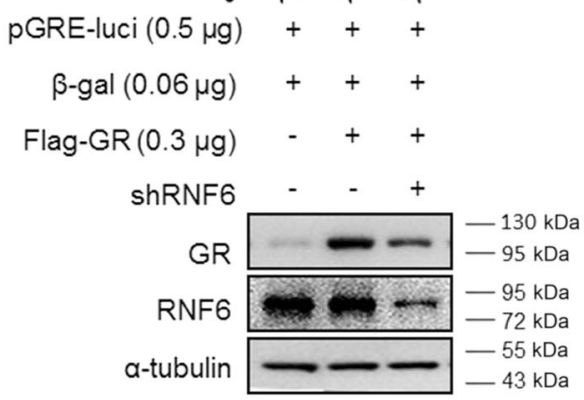

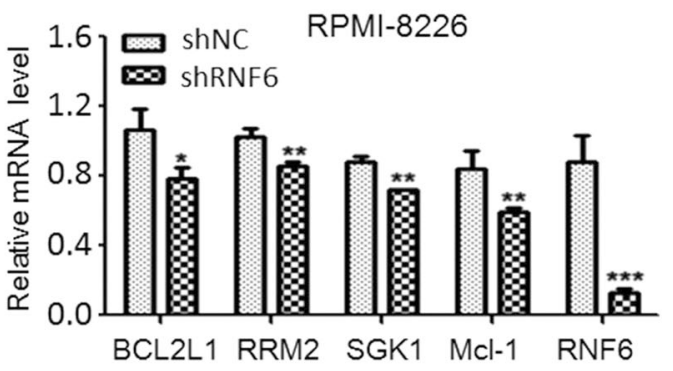

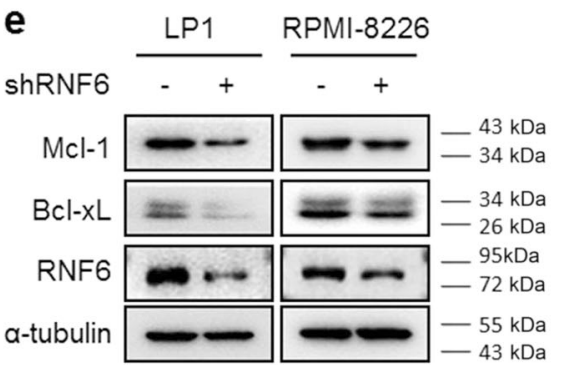

Fig. 5 RNF6 promotes GR activity and the expression of prosurvival genes in MM cells. pGRE, $\beta$-gal, GR, and Myc-RNF6 (a) or shRNF6 (b) were cotransfected into HEK293T cells. GR transcriptional activity was measured by luciferase assays. The same lysates were used to measure the indicated protein level using IB assays. c LP1 and RPMI-8226 cells were infected with RNF6 shRNA, followed by total RNA extraction and qRTPCR to determine the levels of specific genes as indicated. d LP1 and RPMI-8226 cells were infected with increasing amounts of pLVX-RNF6 lentivirus for $72 \mathrm{~h}$. The cells were then subjected to IB assays to determine the expression of Mcl-1 and Bcl-xL. e LP1 and RPMI-8226 cells were infected with shNC or shRNF6 for $96 \mathrm{~h}$, followed by IB assays against specific proteins as indicated. $\mathbf{f}$ LP1 and RPMI-8226 cells transfected with or without siGR were infected with pLVX-RNF6 lentivirus. Cells were subjected to IB assays to determine the expression of Mcl-1 and Bcl-xL. ${ }^{*} P<0.05,{ }^{* *} P<0.01,{ }^{* *} P<0.001$. 

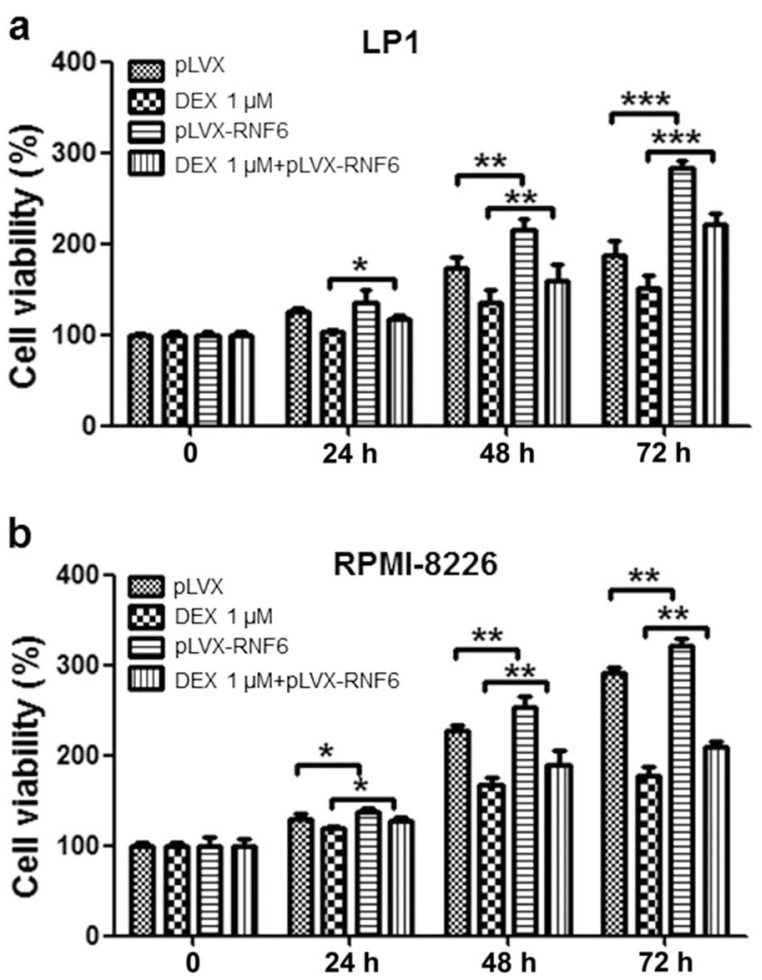

Fig. 6 RNF6 partly prevents the DEX-induced decrease in MM cell proliferation. a LP1 and b RPMI-8226 cells infected with pLVX-RNF6 lentivirus were treated with dexamethasone (DEX), followed by MTT assays after the indicated incubation periods

and confers resistance to DEX. A mechanistic investigation revealed that RNF6 interacts with GR, induces its K63-linked polyubiquitination, increases GR stability and transcriptional activity, and finally promotes MM cell proliferation.

$\mathrm{GR}$, a typical nuclear receptor, is essential for a broad spectrum of biological and pathophysiological activities upon stimulation by its specific factors, including stress, osteoporosis, inflammation, and cancer, via both genomic and nongenomic pathways [21, 22]. Targeting the GR pathway is a major strategy for the treatment of MM; however, resistance to dexamethasone is frequently observed in patients with advanced MM. The molecular events contributing to this resistance include (1) functional defects in GR; (2) increased secretion of prosurvival cytokines in the BM microenvironment; (3) genetic abnormalities; and (4) the DNA methylation and histone modification of genes, especially tumor suppressors [23]. Among all these factors, the loss of GR expression has been most frequently investigated. However, some GRexpressing MM cells such as U266 cells, a typical MM cell line available from ATCC, have also been found to be resistant to dexamethasone $[24,25]$. The U266 cell line was found to express a high level of GR (Fig. $3 \mathrm{~g}$ ); however, it is highly resistant to dexamethasone $[24,25]$. These findings suggest that other regulatory factors, such as PTMs, also alter GR function. Previous studies have found that GR can undergo various PTMs including phosphorylation, SUMOylation, acetylation, nitrosylation, and ubiquitination [19]. For example, acetylation of GR within the hinge region can repress its transcriptional activity [26], while polyubiquitination of GR at K426 can lead to its degradation in proteasomes, thereby suppressing its activity [27].

The present study found that RNF6 might be a novel factor contributing to MM resistance to dexamethasone. RNF6, a ubiquitin ligase, can induce the attachment of K63-linked polyubiquitin chains to $G R$ that is believed to be nondegradable. RNF6 has been found to target SHP-1 and TLE3 for polyubiquitination and subsequent degradation in colorectal cancer cells $[3,4]$. To our surprise, our present study showed that RNF6 prevents $G R$ degradation and that $G R$ thus accumulates in a time- and concentration-dependent manner. Although the detailed mechanisms of this effect remain to be further investigated, RNF6 was also found to stabilize AR and ER, two other nuclear proteins, in prostate cancer and breast cancer, respectively $[2,17]$. To our surprise, RNF6 was also shown to stabilize these nuclear receptors in a manner independent of its ubiquitin ligase activity; when the RING domain was deleted, RNF6 6 RING retained its ability to prevent $G R, A R$, and ER degradation $[2,17]$. These findings suggest that RNF6 is an important factor in maintaining and stabilizing these nuclear receptors that therefore promotes their oncogenic transcriptional activities.

GR activation regulates gene transcription by binding to specific GREs upstream of target genes, thus modulating their transcription. Glucocorticoids are important stimulators of GR activation by binding to the LBD of GR. However, the physiology and pathophysiology of GR might vary in specific contexts in various cancers. For example, dexamethasone can promote breast cancer cell survival and metastasis $[28,29]$ but induces apoptosis in MM and leukemia cells $[24,30,31]$. Global gene expression analyses indicate that more than 1300 genes are induced or repressed by glucocorticoids via GR in liver tissues [32] and that more than 900 genes are modulated in MM cells [9]. GR can promote MM cell proliferation and survival through regulating these genes, such as BCL2L1, RRM2 [9], SGK1 [33], and MCl-1 [34]. It has also been reported that dexamethasone can inhibit apoptosis in fibrosarcomas by transcriptionally activating $\mathrm{BCl}-\mathrm{xL}$ [21]. In the present study, we found that RNF6 promotes GR transcriptional activity and upregulates the expression of $\mathrm{Bcl}-\mathrm{xL}$ and $\mathrm{Mcl}-1$ through regulating GR because RNF6 failed to induce the expression of these two genes when GR was silenced by specific siRNA. Consistent with this hypothesis, downregulation of RNF6 decreased the expression of these genes and eventually led to the apoptosis of MM cells. Notably, RNF6 was also shown to bind to GR at the LBD of GR, and this binding probably activates GR but might also activate the oncogenic transcriptional activity of GR, thus upregulating the transcription of prosurvival genes such as $\mathrm{Bcl}-$ $\mathrm{xL}$ and $\mathrm{Mcl}-1$.

Taken together, the results of the present study highlight a novel mechanism by which GR stability and transcriptional activity are modulated in MM. RNF6, a ubiquitin ligase, promotes GR stability and transcriptional activity by inducing its K63-linked polyubiquitination. Knockdown of RNF6 inhibits cell viability and induces cell apoptosis. All these findings establish a framework for pharmacologically targeting RNF6 as a therapeutic strategy for MM.

\section{ACKNOWLEDGEMENTS}

This work was supported by the National Natural Science Foundation of China (81770154 and 81320108023 to XLM; 81873443 to XWT), the Natural Science Foundation of Jiangsu Higher Education Institutes of China (17KJA180010 to XLM; 19KJA210002 to XWT), Frontier Clinical Technical Project of the Science and Technology Department of Jiangsu Province (BE2017655 to XWT), The Jiangsu Provinicial Medical Talent (ZDRCA2016045 to XWT), and the Priority Academic Program Development of Jiangsu Higher Education Institutions (PAPD).

\section{AUTHOR CONTRIBUTIONS}

XLM designed the study; YR, XX, CYM, KKH, YJX, BYC, and ZBZ conducted experiments; $\mathrm{XLM}, \mathrm{YR}, \mathrm{XX}$, and GS analyzed data; $\mathrm{XWT}$ provided key reagents and clinical data analysis; and XLM and YR wrote the manuscript. 


\section{ADDITIONAL INFORMATION}

Competing interests: The authors declare no competing interests.

\section{REFERENCES}

1. Uchida C, Kitagawa M. RING-, HECT-, and RBR-type E3 Ubiquitin Ligases: involvement in human cancer. Curr Cancer Drug Targets. 2016;16:157-74.

2. Xu K, Shimelis H, Linn DE, Jiang R, Yang $X$, Sun F, et al. Regulation of androgen receptor transcriptional activity and specificity by RNF6-induced ubiquitination. Cancer Cell. 2009;15:270-82.

3. Liu L, Zhang Y, Wong CC, Zhang J, Dong Y, Li X, et al. RNF6 promotes colorectal cancer by activating the Wnt/beta-catenin pathway via ubiquitination of TLE3. Cancer Res. 2018;78:1958-71.

4. Liang Q, Ma D, Zhu X, Wang Z, Sun TT, Shen C, et al. RING-finger protein 6 amplification activates JAK/STAT3 pathway by modifying SHP-1 ubiquitylation and associates with poor outcome in colorectal cancer. Clin Cancer Res. 2018;24:1473-85.

5. Xu X, Han $\mathrm{K}$, Tang $\mathrm{X}$, Zeng $\mathrm{Y}$, Lin $\mathrm{X}$, Zhao $\mathrm{Y}$, et al. The ring finger protein RNF6 induces leukemia cell proliferation as a direct target of pre-B-cell leukemia homeobox 1. J Biol Chem. 2016;291:9617-28.

6. Lu Q, He Y, Wang Y, Gao L, Zheng Y, Zhang Z, et al. Saponins from Paris forrestii (takht.) H. Li display potent activity against acute myeloid leukemia by suppressing the RNF6/AKT/mTOR signaling pathway. Front Pharmacol. 2018;9:673.

7. Ploner C, Schmidt S, Presul E, Renner K, Schrocksnadel K, Rainer J, et al. Glucocorticoid-induced apoptosis and glucocorticoid resistance in acute lymphoblastic leukemia. J Steroid Biochem Mol Biol. 2005;93:153-60.

8. Schmidt S, Irving JA, Minto L, Matheson E, Nicholson L, Ploner A, et al. Glucocorticoid resistance in two key models of acute lymphoblastic leukemia occurs at the level of the glucocorticoid receptor. FASEB J. 2006;20:2600-2.

9. Thomas AL, Coarfa C, Qian J, Wilkerson JJ, Rajapakshe K, Krett NL, et al. Identification of potential glucocorticoid receptor therapeutic targets in multiple myeloma. Nucl Recept Signal. 2015;13:e006.

10. Anbalagan M, Huderson B, Murphy L, Rowan BG. Post-translational modifications of nuclear receptors and human disease. Nucl Recept Signal. 2012;10:e001.

11. Hua G, Paulen L, Chambon P. GR SUMOylation and formation of an SUMO-SMRT/ NCoR1-HDAC3 repressing complex is mandatory for GC-induced IR nGREmediated transrepression. Proc Natl Acad Sci USA 2016;113:E626-34.

12. Duma $D$, Jewell $C M$, Cidlowski JA. Multiple glucocorticoid receptor isoforms and mechanisms of post-translational modification. J Steroid Biochem Mol Biol. 2006;102:11-21.

13. Wang X, DeFranco DB. Alternative effects of the ubiquitin-proteasome pathway on glucocorticoid receptor down-regulation and transactivation are mediated by CHIP, an E3 ligase. Mol Endocrinol. 2005;19:1474-82.

14. Mao X, Hou T, Cao B, Wang W, Li Z, Chen S, et al. The tricyclic antidepressant amitriptyline inhibits $\mathrm{D}$-cyclin transactivation and induces myeloma cell apoptosis by inhibiting histone deacetylases: in vitro and in silico evidence. Mol Pharmacol. 2011;79:672-80.

15. Wang S, Juan J, Zhang Z, Du Y, Xu Y, Tong J, et al. Inhibition of the deubiquitinase USP5 leads to c-Maf protein degradation and myeloma cell apoptosis. Cell Death Dis. 2017;8:e3058.
16. Xu Y, Zhang Z, Li J, Tong J, Cao B, Taylor $P$, et al. The ubiquitin-conjugating enzyme UBE2O modulates c-Maf stability and induces myeloma cell apoptosis. J Hematol Oncol. 2017;10:132.

17. Zeng Y, Xu X, Wang S, Zhang Z, Liu Y, Han K, et al. Ring finger protein 6 promotes breast cancer cell proliferation by stabilizing estrogen receptor alpha. Oncotarget. 2017;8:20103-12.

18. Tursun B, Schluter A, Peters MA, Viehweger B, Ostendorff HP, Soosairajah J, et al The ubiquitin ligase Rnf6 regulates local LIM kinase 1 levels in axonal growth cones. Genes Dev. 2005;19:2307-19.

19. Vandevyver $S$, Dejager $L$, Libert $C$. Comprehensive overview of the structure and regulation of the glucocorticoid receptor. Endocr Rev. 2014;35:671-93.

20. Chauhan D, Auclair D, Robinson EK, Hideshima T, Li G, Podar K, et al. Identification of genes regulated by dexamethasone in multiple myeloma cells using oligonucleotide arrays. Oncogene. 2002;21:1346-58.

21. Gascoyne DM, Kypta RM, Vivanco M. Glucocorticoids inhibit apoptosis during fibrosarcoma development by transcriptionally activating $\mathrm{BCl}-\mathrm{xL}$. J Biol Chem. 2003;278:18022-9.

22. Oakley RH, Cidlowski JA. The biology of the glucocorticoid receptor: new signaling mechanisms in health and disease. J Allergy Clin Immunol. 2013;132:1033-44.

23. Robak P, Drozdz I, Szemraj J, Robak T. Drug resistance in multiple myeloma. Cancer Treat Rev. 2018;70:199-208.

24. Sharma S, Lichtenstein A. Dexamethasone-induced apoptotic mechanisms in myeloma cells investigated by analysis of mutant glucocorticoid receptors. Blood. 2008;112:1338-45.

25. Mao X, Stewart AK, Hurren R, Datti A, Zhu X, Zhu Y, et al. A chemical biology screen identifies glucocorticoids that regulate c-maf expression by increasing its proteasomal degradation through up-regulation of ubiquitin. Blood. 2007;110:4047-54.

26. Kino T, Chrousos GP. Acetylation-mediated epigenetic regulation of glucocorticoid receptor activity: circadian rhythm-associated alterations of glucocorticoid actions in target tissues. Mol Cell Endocrinol. 2011;336:23-30.

27. Wallace AD, Cidlowski JA. Proteasome-mediated glucocorticoid receptor degradation restricts transcriptional signaling by glucocorticoids. J Biol Chem. 2001;276:42714-21.

28. Li Z, Dong J, Zou T, Du C, Li S, Chen C, et al. Dexamethasone induces docetaxel and cisplatin resistance partially through up-regulating Kruppel-like factor 5 in triple-negative breast cancer. Oncotarget. 2017;8:11555-65.

29. Obradovic MMS, Hamelin B, Manevski N, Couto JP, Sethi A, Coissieux MM, et al. Glucocorticoids promote breast cancer metastasis. Nature. 2019;567:540-4.

30. Smith LK, Cidlowski JA. Glucocorticoid-induced apoptosis of healthy and malignant lymphocytes. Prog Brain Res. 2010;182:1-30.

31. Frankfurt O, Rosen ST. Mechanisms of glucocorticoid-induced apoptosis in hematologic malignancies: updates. Curr Opin Oncol. 2004;16:553-63.

32. Phuc LeP, Friedman JR, Schug J, Brestelli JE, Parker JB, Bochkis IM, et al. Glucocorticoid receptor-dependent gene regulatory networks. PLoS Genet. 2005;1:e16

33. Fagerli UM, Ullrich K, Stuhmer T, Holien T, Kochert K, Holt RU, et al. Serum/ glucocorticoid-regulated kinase 1 (SGK1) is a prominent target gene of the transcriptional response to cytokines in multiple myeloma and supports the growth of myeloma cells. Oncogene. 2011;30:3198-206.

34. Derenne S, Monia B, Dean NM, Taylor JK, Rapp MJ, Harousseau JL, et al. Antisense strategy shows that $\mathrm{Mcl}-1$ rather than $\mathrm{Bcl}-2$ or $\mathrm{Bcl}-\mathrm{x}(\mathrm{L})$ is an essential survival protein of human myeloma cells. Blood. 2002;100:194-9. 